\title{
CARE-compliant stereotactic radiotherapy of urothelial nodal metastases: A case report
}

\author{
FEDERICA MEDICI ${ }^{1,2}$, GIAMBATTISTA SIEPE ${ }^{2}$, LIDIA STRIGARI ${ }^{3}$, FRANCESCO MASSARI ${ }^{4}$, \\ MILLY BUWENGE ${ }^{1,2}$, SILVIA BISELLO ${ }^{1,2}$, PAOLO CASTELLUCCI ${ }^{5}$, STEFANO FANTI $^{5}$, \\ SILVIA CAMMELLI ${ }^{1,2}$ and ALESSIO GIUSEPPE MORGANTI ${ }^{1,2}$ \\ ${ }^{1}$ Radiation Oncology Unit, Department of Experimental Diagnostic and Specialty Medicine (DIMES), \\ Alma Mater Studiorum Bologna University; ${ }^{2}$ Radiation Oncology Unit, ${ }^{3}$ Medical Physics Unit, \\ ${ }^{4}$ Medical Oncology Unit and ${ }^{5}$ Nuclear Medicine Unit, Istituto di Ricovero e Cura a Carattere \\ Scientifico (IRCCS) Azienda Ospedaliero-Universitaria di Bologna, I-40138 Bologna, Italy
}

Received October 30, 2021; Accepted January 13, 2022

DOI: $10.3892 / \mathrm{mco} .2022 .2518$

\begin{abstract}
The aim of the present study was to report the case of a 58-year-old male patient with ureteral carcinoma who underwent ureteroileostomy treatment. At 2 years following surgery, six lymph node metastases (LNMs) were detected in the patient's para-aortic and pelvic regions using ${ }^{18} \mathrm{~F}$-labeled fluoro-2-deoxyglucose (FDG) positron emission tomography (PET)/CT. All LNMs were treated using stereotactic body radiotherapy (SBRT; 35-40 Gy/5 fractions). At 3 months after radiotherapy, ${ }^{18} \mathrm{~F}-\mathrm{FDG}-\mathrm{PET} / \mathrm{CT}$ examination revealed a complete radiological and metabolic response of all targeted treatment sites in the patient. In the 2 years following radiotherapy, another three same-dose SBRT treatments were performed on single or multiple LNMs, which were all detected in the abdomen and pelvis of the patient. Overall, a total of 11 LNMs were targeted in the patient and all exhibited complete radiological and metabolic response following treatment. The only treatment side effect reported by the patient was a slight and temporary loss of appetite. In patients with lymph node oligometastases there are two options for radiotherapy: i) Irradiation focusing on LNMs alone; and ii) prophylactic irradiation of the entire lymph node area combined with a boost on macroscopic lesions. In the patient discussed in the present study, the choice of irradiation focusing on LNMs alone made
\end{abstract}

Correspondence to: Dr Federica Medici, Radiation Oncology Unit, Department of Experimental Diagnostic and Specialty Medicine (DIMES), Alma Mater Studiorum Bologna University, Via Albertoni 15, I-40138 Bologna, Italy

E-mail: federica.medici4@gmail.com

Abbreviations: LNMs, lymph node metastases; SBRT, stereotactic body radiotherapy; UC, urothelial carcinomas

Key words: case report, urothelial neoplasms, stereotactic radiotherapy, lymph nodes, oligorecurrent it possible to postpone systemic therapies and instead use an optimally tolerated treatment. The treatment outcome in this patient indicated that there was no radioresistance of urothelial LNMs.

\section{Introduction}

Urothelial carcinoma (UC) is the fourth most common malignant tumor. Upper urinary tract UC is rare and accounts for only 5.0-10\% of all diagnosed UCs in Europe (1). The lifetime risk of developing UC in developed countries is 3.9 and $1.2 \%$ in men and women, respectively (2). Additionally, the majority of patients diagnosed with UC are $>70$ years of age (1).

Approximately $4 \%$ of patients with UC display metastasis at diagnosis and $~ 50 \%$ of patients with UC undergoing surgery develop tumor recurrence during subsequent follow-up examinations, with 70-90\% of relapses represented by distant failure (3). In patients with distant failure, there are few therapeutic options available and systemic therapies are currently the first-line treatment option (3). However, some retrospective studies have suggested the possibility of an improved outcome following metastasectomy in selected patients with oligometastatic disease (4-7). Surgical resection of the metastatic site is not always feasible, either due to technical issues or the presence of any concomitant comorbidities (3).

A non-surgical approach based on stereotactic body radiotherapy (SBRT) may be proposed in patients when surgical resection is not feasible, given the efficacy and tolerability of this irradiation technique. In particular, lymph node metastases (LNMs) represent an ideal target for SBRT. The small volume, minimum organ motion, regular shape and well-defined contours make LNMs particularly suitable for treatment using SBRT (8). However, data availability on the effects of SBRT in patients with oligometastatic UC is limited.

The current study reports a case of a patient with recurrent UC who was successfully treated with repeated courses of SBRT on multiple and subsequent metachronous LNMs. The current case report follows the CARE (CAse REports) guidelines (9). 


\section{Case report}

The current study reports a case of a 58-year-old male patient with UC who was referred to our institution. A physical examination and blood chemistry results demonstrated that the patient exhibited no significant abnormalities. Additionally, the patient did not report any neoplastic family history or relevant comorbidities. The patient underwent ureteroileostomy in September 2016 and pathological examination indicated a pT4N2Mx (7 out of 11 resected regional lymph nodes were metastatic) high-grade (G3) UC based on the TNM (Union for International Cancer Control; 8th edition) (10) and WHO/ISUP 2004-2016 grading systems (11), respectively. Based on the histological examination performed on the patient, adjuvant therapy was undertaken (atezolizumab; 1,200 mg every 21 days; 16 cycles).

No local or distant relapse was observed in the patient at subsequent follow-up checks until August 2018. A whole-body ${ }^{18}$ F-labeled fluoro-2-deoxyglucose (FDG) positron emission tomography (PET)/CT scan indicated six LNMs in the para-aortic (2 LNMs), right common iliac (2 LNMs) and presacral (2 LNMs) regions. All detected LNMs were treated with SBRT and the prescribed dose was 35-40 Gy in five daily fractions, depending on the dose delivered to the organs at risk. A subsequent ${ }^{18} \mathrm{~F}-\mathrm{FDG}-\mathrm{PET} / \mathrm{CT}$ scan, which was performed in December 2018, indicated a complete radiological and metabolic response of all treated sites.

In November 2019, a subsequent ${ }^{18}{ }^{\mathrm{F}}$ FDG-PET/CT scan revealed a LNM in the pre-aortic region, which was treated with SBRT (40 Gy in five fractions). In January 2020, the ${ }^{18} \mathrm{~F}-\mathrm{FDG}-\mathrm{PET} / \mathrm{CT}$ scan indicated a complete response of this lesion. In April 2020, a ${ }^{18} \mathrm{~F}-\mathrm{FDG}-\mathrm{PET} / \mathrm{CT}$ indicated a new LNM in the left obturatory lymph node region. Additionally, this LNM was treated with SBRT (40 Gy in five fractions) and the subsequent ${ }^{18} \mathrm{~F}-\mathrm{FDG}-\mathrm{PET} / \mathrm{CT}$ (performed in June 2020) indicated a complete response of this LNM. Furthermore, the latter ${ }^{18} \mathrm{~F}-\mathrm{FDG}-\mathrm{PET} / \mathrm{CT}$ also indicated further LNMs in the interaortocaval lymph nodes and on two left internal iliac lymph nodes, all of which were treated with SBRT (35 and 40 Gy in five fractions, respectively). A ${ }^{18} \mathrm{~F}-\mathrm{FDG}$-PET/CT was performed in October 2020 and indicated a complete radiological and metabolic response in the aforementioned LNMs. The last follow-up performed in December 2020 confirmed a complete response with no evidence of any relapse in the patient.

In the case discussed in the current study, a total of 11 LNMs were irradiated over a period of 22 months, which led to a complete radiological and metabolic response of all lesions without systemic treatment. The anatomical site of the treated LNMs is presented in Fig. 1. In April 2020, during the irradiation of an obturator LNM, the patient reported mild anorexia and dyspnea, which resolved without the need for supportive therapies. Additionally, no severe toxicity was reported. Written informed consent was obtained from the patient for the publication of the current case report.

The timeline of events in this case were as follows: i) Ureteroileostomy (Bricker) of high-grade (G3; WHO 2004-2016) urothelial carcinoma, pT4N2M0, was performed on 2016-09-01; ii) adjuvant therapy (atezolizumab;
16 cycles) was completed on 2017-09-27; iii) on 2018-07-29, a chest-abdomen-pelvis contrast-enhanced CT was performed and indicated enlarged presacral lymph nodes; iv) on 2018-08-14, a multidisciplinary evaluation was performed and SBRT was planned on the oligometastatic sites; v) on 2018-08-24, ${ }^{18 F-F D G-P E T / C T ~ i n d i c a t e d ~ p a t h o l o g i c a l ~ e n h a n c e-~}$ ment in six lymph nodes [two in the paraortic region (SUV 8.4), two in the right common iliac region (SUV 6.8) and two in the presacral region (SUV 8.4 and 2.8)]; vi) on 2018-09-11, SBRT was performed on para-aortic nodes (40 Gy/5 fractions), right common iliac nodes ( $35 \mathrm{~Gy} / 5$ fractions) and presacral nodes (40 Gy/5 fractions); vii) on 2018-12-03, a ${ }^{18} \mathrm{~F}-\mathrm{FDG}-\mathrm{PET} / \mathrm{CT}$ indicated a complete radiological and metabolic response of all treated sites; viii) on 2019-11-08, a chest-abdomen-pelvis contrast enhanced CT indicated nodal oligorecurrence at preaortic/iliac bifurcation; ix) on 2019-11-21, a ${ }^{18} \mathrm{~F}-\mathrm{FDG}-\mathrm{PET} / \mathrm{CT}$ indicated recurrence in a preaortic/iliac bifurcation lymph node and three lymph nodes in the left obturator region and left internal iliac region; $x$ ) on 2019-11-28, SBRT was performed on the latter sites at $40 \mathrm{~Gy} / 5$ fractions; xi) on $2020-01-23, a{ }^{18} \mathrm{~F}-\mathrm{FDG}-\mathrm{PET} / \mathrm{CT}$ indicated a complete radiological response of the treated sites (Fig. 2); xii) on 2020-04-17, a ${ }^{18}$ F-FDG-PET/CT indicated left obturator nodal oligorecurrence; xiii) on 2020-05-06, SBRT was performed on the latter site at $40 \mathrm{~Gy} / 5$ fractions; xiv) on 2020-06-30, a ${ }^{18} \mathrm{~F}-\mathrm{FDG}-\mathrm{PET} / \mathrm{CT}$ indicated one interaortocaval and two left internal iliac nodal oligometastases; xv) on 2020-07-13, SBRT was performed on the interaortocaval lymph node $(35 \mathrm{~Gy} / 5$ fractions) and left internal iliac lymph nodes ( $40 \mathrm{~Gy} / 5$ fractions); $\mathrm{xvi}$ ) on 2020-10-05, a ${ }^{18} \mathrm{~F}-\mathrm{FDG}-\mathrm{PET} / \mathrm{CT}$ indicated a complete radiological and metabolic response of the treated lesions; and xvii) on 2020-12-15 and at the last follow-up, all treated lesions exhibited complete metabolic and radiological response with no evidence of relapse.

\section{Discussion}

The current study reported a 58-year old male patient exhibiting multiple and recurrent LNMs from UC and undergoing repeated SBRT, with tumor control lasting up to 2 years without the use of invasive or systemic therapies. The aforementioned patient case is original for a number of different reasons. Firstly, isolated LNMs are rare in UCs (12). In the patient discussed in the present study, the tumor only recurred in the nodes for an extended period of time. Furthermore, the patient underwent SBRT despite not being fully classified as oligometastatic, as only patients with $1-5$ metastases are traditionally defined as oligometastatic (13). However, considering the excellent general condition of this patient, their willingness to postpone systemic therapy and the fact that the tumor was spreading only to the lymph nodes, which is a condition known for its favorable prognosis, the multidisciplinary team selected SBRT treatment for this case (12). It may be observed that all subsequent disease relapses in this patient may be defined as oligorecurrences, as the number of new LNMs was $<5$. The data collected in the current study suggest that there can be some flexibility in the selection of patients referred for SBRT. Furthermore, previous data have indicated that SBRT is less effective in UCs compared with other cancer types and in terms of local control $(14,15)$. However, all 11 lesions treated in the current case had a complete 


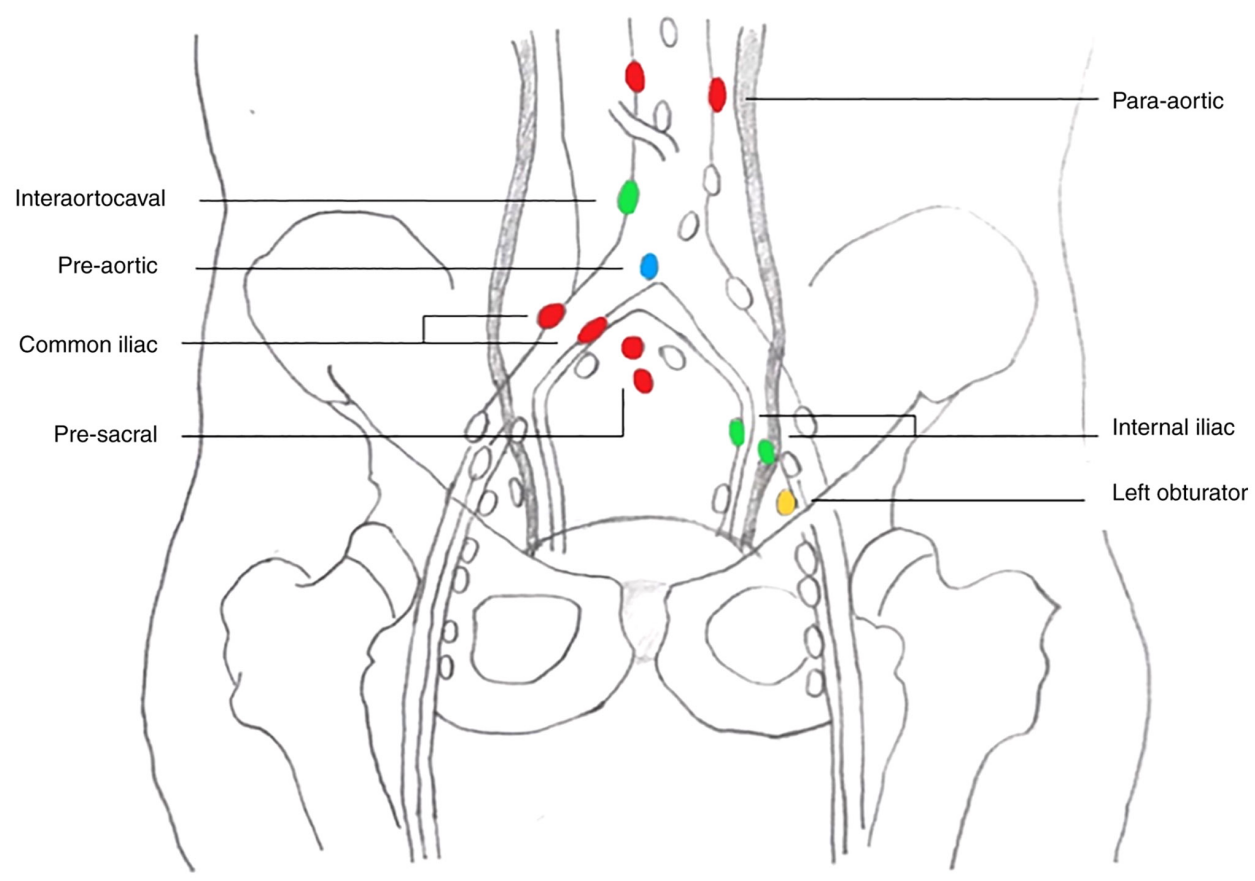

Figure 1. Nodal metastases irradiated in September 2018 are indicated in red. Two para-aortic, two right common iliac and two presacral nodal metastases were identified. Nodal metastases irradiated in November 2019 are indicated in blue. One preaortic/iliac bifurcation node was identified. Nodal metastasis irradiated in May 2020 are indicated in yellow. One left obturatory node was identified. Nodal metastases irradiated in July 2020 are indicated in green. One interaortocaval and two left internal iliac nodes were identified.
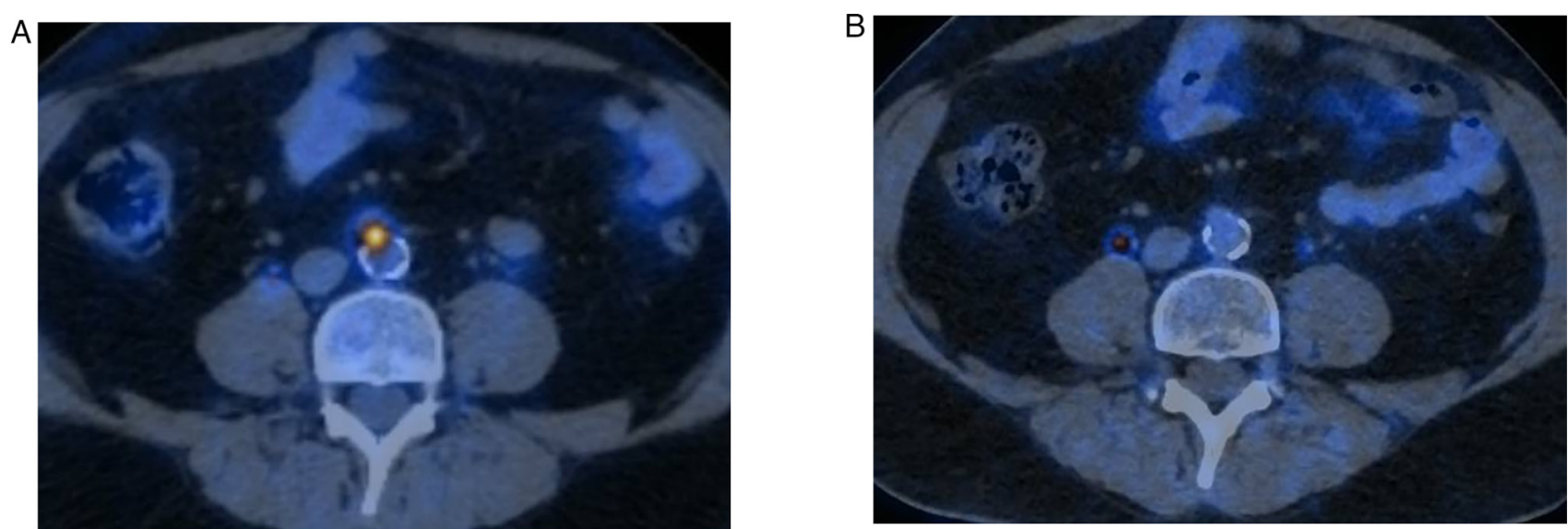

Figure 2. (A) 18F-FDG-PET/CT performed in November 2019 demonstrated recurrence in a preaortic/iliac bifurcation lymph node. (B) 18F-FDG-PET/CT performed in January 2020 after stereotactic body radiotherapy on the latter site showing complete radiological response. $18 \mathrm{~F}-\mathrm{FDG}-\mathrm{PET} / \mathrm{CT}$, ${ }^{18} \mathrm{~F}-\mathrm{labeled}$ fluoro-2-deoxyglucose positron emission tomography.

response following SBRT, confirming the effectiveness of this technique in the treatment of LNMs (8).

The current case report highlights a relevant issue in the treatment of lymph node oligometastases. A well-known problem following therapies that focus only on macroscopic metastatic sites is the risk of further regional LNMs (8). The risk of this is estimated to be $21.4 \%$ (range, $10.5-58.0 \%$ ) following SBRT, which is a figure that is based on a small amount of data (16). A number of different strategies have been proposed to address this problem. Firstly, SBRT may be used as a boost on macroscopic LNMs following regional prophylactic irradiation. For example, Seo et al (17) reported significantly higher disease control on regional nodes in patients treated with external beam radiotherapy on the paraaortic region followed by SBRT boost compared with patients treated with SBRT alone (5-year regional control: $92 \%$ vs. $34 \% ; \mathrm{P}=0.006$ ). The first tumor recurrence in the patient discussed in the present case study involved pelvic and abdominal lymph nodes. Therefore, it may be suggested that a prophylactic treatment of the pelvic lymph nodes that was extended to the para-aortic region and associated with a SBRT-boost on LNMs may have led to avoidance of subsequent relapses, which all occurred in the same areas. Another strategy to reduce the risk of further regional LNMs is less extensive prophylactic irradiation, with SBRT at 'adjuvant' doses delivered to the macroscopic disease and to the 
contiguous part of the lymphatic pathway, combined with a high dose-simultaneous integrated boost to the macroscopic LNM only (18). However, the results of this irradiation strategy are not fully elucidated in terms of improved regional control. Furthermore, it should be noted that the aforementioned technique would not have been useful in the patient outlined in the current study as all new relapses occurred at some distance from treated sites (Fig. 1).

In the case of the patient discussed in the current study, SBRT was used in order to postpone the start of systemic therapy. However, it should be noted that this technique was used for a variety of therapeutic purposes, including the local consolidation of metastatic lesions treated with systemic therapy and the local control of oligoprogressive lesions during the course of systemic therapy, in order to postpone the initiation of subsequent treatment lines $(17,19)$. The choice between these treatments may depend on a number of varied factors, such as the presence of primary cancer, availability of effective systemic therapies and patient preferences. In the case outlined in the current study, the patient's initial willingness to avoid chemotherapy was considered a priority.

In conclusion, the case reported in the present study suggests the possibility of achieving a complete response in subsequent metachronous LNMs from UC treated with SBRT. Further studies on this subject should aim to investigate the following: i) Multicenter databases to evaluate the SBRT impact on both local control and progression-free survival in a large dataset; ii) the comparison of strategies based on SBRT combined with systemic treatments with strategies based on SBRT alone followed by systemic treatments only in case of widespread metastases; iii) the most effective combination of systemic therapies and SBRT in terms of timing, types of drugs and SBRT dose/fractionation regimens. Given the theoretical advantages of a SBRT-immunotherapy combination treatment, it may be noted that a recent study has indicated the tolerability of the SBRT-pembrolizumab combination in patients with UC (20).

\section{Acknowledgements}

Not applicable.

\section{Funding}

No funding was received.

\section{Availability of data and materials}

All data generated or analyzed during this study are included in this published article.

\section{Authors' contributions}

All authors conceptualized the study. FM, GS and SB wrote the manuscript and prepared the original draft. MB, SC, PC and AGM reviewed and edited the manuscript. LS, SF, FM and AGM supervised the study. FM and AGM confirm the authenticity of all the raw data. All authors have read and approved the final manuscript.

\section{Ethics approval and consent to participate}

Not applicable.

\section{Patient consent for publication}

The patient has been correctly informed and provided written informed consent for the publication of any associated data and accompanying images.

\section{Competing interests}

The authors declare that they have no competing interests.

\section{References}

1. Rouprêt M, Babjuk M, Compérat E, Zigeuner R, Sylvester RJ, Burger M, Cowan NC, Böhle A, Van Rhijn BW, Kaasinen E, et al: European association of urology guidelines on upper urinary tract urothelial cell carcinoma: 2015 update. Eur Urol 68: 868-879, 2015.

2. Noone AM, Howlader N, Krapcho M, Miller D, Brest A, Yu M, Ruhl J, Tatalovich Z, Mariotto A, Lewis DR, et al (eds): SEER Cancer Statistics Review 1975-2015. National Cancer Institute, Bethesda, MD, 2018.

3. Spiess PE, Agarwal N, Bangs R, Boorjian SA, Buyyounouski MK, Clark PE, Downs TM, Efstathiou JA, Flaig TW, Friedlander T, et al: Bladder Cancer, Version 5.2017, NCCN Clinical Practice Guidelines in Oncology. J Natl Compr Canc Netw 15: 1240-1267, 2017.

4. Siefker-Radtke AO, Walsh GL, Pisters LL, Shen Y, Swanson DA, Logothetis CJ and Millikan RE: Is there a role for surgery in the management of metastatic urothelial cancer? The M. D. Anderson experience. J Urol 171: 145-148, 2004.

5. Lehmann J, Suttmann H, Albers P, Volkmer B, Gschwend JE, Fechner G, Spahn M, Heidenreich A, Odenthal A, Seif C, et al: Surgery for metastatic urothelial carcinoma with curative intent: The German experience (AUO AB 30/05). Eur Urol 55: 1293-1299, 2009.

6. Dodd PM, McCaffrey JA, Herr H, Mazumdar M, Bacik J, Higgins G, Boyle MG, Scher HI and Bajorin DF: Outcome of postchemotherapy surgery after treatment with methotrexate, vinblastine, doxorubicin, and cisplatin in patients with unresectable or metastatic transitional cell carcinoma. J Clin Oncol 17: 2546-2552, 1999.

7. Abe T, Shinohara N, Harabayashi T, Sazawa A, Maruyama S, Suzuki S and Nonomura K: Impact of multimodal treatment on survival in patients with metastatic urothelial cancer. Eur Urol 52: 1106-1113, 2007.

8. Deodato F, Ferro M, Cilla S, Ianiro A, Buwenge M, Re A, Sallustio G, Valentini V, Morganti AG and Macchia G: Stereobody radiotherapy for nodal recurrences in oligometastatic patients: A pooled analysis from two phase I clinical trials. Clin Exp Metastasis 37: 519-529, 2020.

9. Riley DS, Barber MS, Kienle GS, Aronson JK von Schoen-Angerer T, Tugwell $\mathrm{P}$, Kiene $\mathrm{H}$, Helfand $\mathrm{M}$, Altman DG, Sox H, et al: CARE guidelines for case reports: Explanation and elaboration document. J Clin Epidemiol 89: 218-235, 2017.

10. Brierley JD, Gospodarowicz MK and Wittekind C (eds): TNM Classification of Malignant Tumours. 8th edition. John Wiley \& Sons, Inc. Hoboken, NJ, pp1-272,2017.

11. Compérat EM, Burger M, Gontero P, Mostafid AH, Palou J, Rouprêt M, van Rhijn BWG, Shariat SF, Sylvester RJ, Zigeuner R and Babjuk M: Grading of urothelial carcinoma and the new 'World health organisation classification of tumours of the urinary system and male genital organs 2016'. Eur Urol Focus 5: 457-466, 2019.

12. Margulis V, Shariat SF, Matin SF, Kamat AM, Zigeuner R, Kikuchi E, Lotan Y, Weizer A, Raman JD and Wood CG; Upper Tract Urothelial Carcinoma CollaborationThe Upper Tract Urothelial Carcinoma Collaboration: Outcomes of radical nephroureterectomy: A series from the Upper Tract Urothelial Carcinoma Collaboration. Cancer 115: 1224-1233, 2009. 
13. Hellman S and Weichselbaum RR: Oligometastases. J Clin Oncol 13: 8-10, 1995.

14. Loi M, Frelinghuysen M, Klass ND, Oomen-De Hoop E Granton PV, Aerts J, Verhoef C and Nuyttens J: Locoregional control and survival after lymph node SBRT in oligometastatic disease. Clin Exp Metastasis 35: 625-633, 2018.

15. Francolini G, Desideri I, Detti B, Di Cataldo V, Masi L, Caramia G, Visani L, Terziani F, Muntoni C, Lo Russo M, et al: Stereotactic radiotherapy in oligoprogressive and oligorecurrent urothelial cancer patients: A retrospective experience. Cancer Treat Res Commun 19: 100124, 2019.

16. Deodato F, Macchia G, Buwenge M, Bonetti M, Cilla S, Zamagni A, Re A, Pezzulla D, Cellini F, Strigari L, et al: Systematic review of stereotactic body radiotherapy for nodal metastases. Clin Exp Metastasis 38: 11-29, 2021

17. Seo YS, Kim MS, Cho CK, Yoo HJ, Jang WI, Kim KB, Lee DH, Moon SM and Lee HR: Stereotactic body radiotherapy for oligometastases confined to the para-aortic region: Clinical outcomes and the significance of radiotherapy field and dose. Cancer Invest 33: 180-187, 2015.
18. Kneebone A, Hruby G, Ainsworth H, Byrne K, Brown C, Guo L, Guminski A and Eade T: Stereotactic body radiotherapy for oligometastatic prostate cancer detected via prostate-specific membrane antigen positron emission tomography. Eur Urol Oncol 1: 531-537, 2018.

19. Detti B, D'Angelillo RM, Ingrosso G, Olmetto E, Francolini G, Triggiani L, Bruni A, Borghesi S, Fondelli S, Carfagno T, et al: Combining abiraterone and radiotherapy in prostate cancer patients who progressed during abiraterone therapy. Anticancer Res 37: 3717-3722, 2017

20. Sundahl N, Vandekerkhove G, Decaestecker K, Meireson A De Visschere P, Fonteyne V, De Maeseneer D, Reynders D, Goetghebeur E, Van Dorpe J, et al: Randomized phase 1 trial of pembrolizumab with sequential versus concomitant stereotactic body radiotherapy in metastatic urothelial carcinoma. Eur Urol 75: 707-711, 2019.

This work is licensed under a Creative Commons Attribution-NonCommercial-NoDerivatives 4.0 International (CC BY-NC-ND 4.0) License. 